\title{
MicroRNA-141 and its associated gene FUS modulate proliferation, migration and cisplatin chemosensitivity in neuroblastoma cell lines
}

\author{
ZIRAN WANG ${ }^{1}$, HONGYAN LEI ${ }^{1}$ and QUANYU SUN ${ }^{2}$ \\ ${ }^{1}$ Emergency Department, and ${ }^{2}$ Department of Medical Imaging, Linyi People's Hospital, \\ Linyi, Shandong 276001, P.R. China
}

Received November 19, 2015; Accepted December 27, 2015

DOI: $10.3892 /$ or.2016.4640

\begin{abstract}
In the present study, a novel signaling pathway of microRNA-141 (miR-141)/fused in sarcoma (FUS) was investigated in neuroblastoma (NB). Gene expression of miR-141 was evaluated in $6 \mathrm{NB}$ cell lines. IMR-32 and SH-SY5Y cells were transduced with the miR-141 mimic lentivirus. The effects of miR-141 upregulation on cell proliferation, cell division, migration, chemosensitivity and in vivo explants were evaluated by MTT, cell cycle, wound-healing, cisplatin sensitivity and in vivo tumor growth assays, respectively. The correlation between miR-141 and the FUS gene was evaluated by luciferase assay and qRT-PCR. FUS was also downregulated in IMR-32 and SH-SY5Y cells to evaluate its impact on NB regulation. miR-141 was downregulated in both $M Y C N$ - and non- $M Y C N$-amplified NB cell lines. In the IMR-32 and SH-SY5Y cells, lentivirus-induced miR-141 upregulation inhibited cancer proliferation, cell cycle progression, migration and increased cisplatin chemosensitivity in vitro. In addition, miR-141 upregulation reduced the in vivo growth of IMR-32 tumor explants. FUS was found to be inversely regulated by miR-141 in NB. Small interfering RNA (siRNA)induced FUS downregulation had similar tumor-suppressive effects as miR-141 upregulation on NB cell proliferation, cell cycle progression, migration and cisplatin chemosensitivity. Our data indicate that miR-141 and the FUS gene, which are inversely correlated, play significant functional roles in regulating human NB.
\end{abstract}

Correspondence to: Dr Quanyu Sun, Department of Medical Imaging, Linyi People's Hospital, 49 Yizhou Road, Linyi, Shandong 276001, P.R. China

E-mail: q_sun@aol.com

Key words: neuroblastoma, miR-141, FUS, proliferation, migration, cisplatin

\section{Introduction}

Neuroblastoma (NB) is one of the most common malignant tumors in children (1). In the US, NB is the third most common cause of cancer-related mortality $(\sim 6 \%)$ among children aged 1-14 years (2). The subtypes of NB show great heterogeneity in regard to genetic abnormalities, which are closely associated with clinical outcomes among NB patients (3). For example, in patients presenting with the $M Y C$-related oncogene $(M Y C N)$ (amplified subtype of NB), the $M Y C N$ gene is aberrantly repeated at chromosome $2 \mathrm{p} 24$, resulting in NB progression to advanced stages, aggressive metastasis and poor patient prognosis (3-5). Unfortunately, the underlying molecular mechanisms contributing to NB pathogenesis, metastasis and apoptosis are large unknown. It, thus, presents a great challenge to identifying novel biomarkers or therapeutic targets for early detection or treatment for young patients with NB.

MicroRNAs (miRNAs) are families of highly conserved non-coding small RNAs that attach to the 3'-untranslated region (3'-UTR) of downstream target genes to post-transcriptionally suppress gene expression, thus regulating various cellular processes in both animals and human diseases (6). miRNAs have been shown to play critical roles in NB pathogenesis, metastasis and apoptosis $(7,8)$. Among many of the oncogenic or tumor-suppressor miRNAs, miR-141 is highly expressed in the circulating plasma in patients with advanced stages of metastatic colon cancer, suggesting an oncogenic role as a colon cancer biomarker (9). In contrast, miR-141 was shown to be downregulated in gastric and prostate cancer, presumably acting as a tumor-suppressor of cancer progression and metastasis $(10,11)$. In NB, although a previous study showed that miR-141 is differentially expressed among NB subtypes (12), the exact expression profiles or mechanisms of miR-141 in NB remain elusive.

The fused in sarcoma (FUS) gene, which encodes an RNA binding protein, is associated with genetic disorders particularly neurodegenerative diseases such as amyotrophic lateral sclerosis (ALS) $(13,14)$. In human prostate cancer, FUS is shown to be a tumor-suppressor gene as its overexpression inhibited cancer growth and promoted cancer apoptosis (15). In a recent study, FUS was reported to be highly expressed in NB SK-N-AS cells (16). However, similar to miR-141, the 
exact expression and function of FUS in human NB are largely unknown.

In the present study, we firstly evaluated the expression of miR-141 in both $M Y C N$ - and non-MYCN-amplified NB cell lines. We then upregulated miR-141 in two NB cell lines IMR-32 and SH-SY5Y in to evaluate the possible tumor-suppressive role of miR-141 on NB proliferation, cell cycle progression, metastasis and chemosensitivity. In addition, we hypothesized that the FUS gene is the downstream target of miR-141, and is thereby inversely associated with miR-141 regulation in NB. To test this hypothesis, we utilized siRNA-mediated FUS downregulation to evaluate its effect on NB proliferation, cell cycle progression, metastasis and chemosensitivity.

\section{Materials and methods}

Cell culture. Three human NB cell lines used in the present study, IMR-32, SH-SY5Y and S-K-NAS, were purchased from the American Type Culture Collection (ATCC; Manassas, VA, USA). Another three human NB cell lines, NB-1691, LAN-5 and LAN-6, were purchased from the Cell Bank of the Type Culture Collection of the Chinese Academy of Sciences (Shanghai, China). The control cell line, human retinal-pigmented epithelial cells immortalized with telomerase reverse transcriptase (hTERT-RPE1) was purchased from Clontech (USA). All cells were maintained in RPMI1640 medium supplemented with $10 \%$ fetal bovine serum and penicillin/streptomycin (pen/strep) (100 U/ml pen and $100 \mathrm{mg} / \mathrm{ml} \mathrm{strep)} \mathrm{(all} \mathrm{from} \mathrm{Invitrogen,} \mathrm{USA)} \mathrm{in} \mathrm{a} \mathrm{humidified}$ chamber with $5 \% \mathrm{CO}_{2}$ at $37^{\circ} \mathrm{C}$.

RNA extraction and quantitative real-time PCR. Total RNA was isolated from the NB cells using an RNeasy Mini kit (Qiagen, USA) according to the manufacturer's protocol. From each sample, $1 \mu \mathrm{g}$ RNA was used for reverse transcriptions using a Transcriptor First Strand cDNA Synthesis kit (Roche, USA) according to the manufacturer's protocol. Quantitative real-time PCR (qRT-PCR) was carried out in two manners. For miR-141 detection, a TaqMan miRNA assay (Applied Biosystems, USA) was carried out with U6 snRNA as endogenous control. For FUS detection, a Brilliant II SYBR-Green qPCR Master Mix (Stratagene, USA) was used with glyceraldehyde-3-phosphate dehydrogenase (GAPDH) as the endogenous control. Relative gene expression levels were calculated using the $2^{-\Delta \Delta \mathrm{Ct}}$ method.

miR-141 overexpression assay. The forced overexpression of miR-141 in NB cell lines was achieved by lentiviral transduction. The miR-141 mimic lentivirus (miR-141-mimics), and its corresponding control miRNA lentivirus (C-miRNA) were purchased from SunBio Medical Biotechnology (Shanghai, China). In the NB culture, IMR-32 and SH-SY5Y cells were transduced with 250 pmol lentiviruses for $48 \mathrm{~h}$ in the presence of $8 \mu \mathrm{g} / \mathrm{ml}$ Polybrene (Sigma-Aldrich, USA) and a multiplicity of infection (MOI) of 20 . Subsequently, the cells were briefly washed with phosphate-buffered saline (PBS) and continuously maintained in fresh culture medium for 48 or $72 \mathrm{~h}$ to stabilize the lentivirus transduction. The floating cells were then removed. The healthy cells were then subject to qRT-PCR examination to verify the efficacy of the overexpression assay.
Proliferation assay. After lentiviral transduction or siRNA transfection, the IMR-32 and SH-SY5Y cells were seeded in 96 -well plates $\left(3.5 \times 10^{3} /\right.$ well $)$ and maintained for 5 days. The proliferation of NB cells was characterized using a 3-(4,5-dimethylthiazol-2-yl)-2,5-diphenyltetrazolium bromide (MTT) assay (Sigma-Aldrich) according to the manufacturer's protocol. Briefly, every $24 \mathrm{~h}$ during the proliferation assay, each well of 96-well plates was added with $200 \mu \mathrm{l}$ MTT for $2 \mathrm{~h}$, then treated with $150 \mu \mathrm{l}$ dimethyl sulfoxide (DMSO) for another $3 \mathrm{~h}$. The optical density at $570 \mathrm{~nm}$ was measured using an ultraviolet-visible spectrophotometer (Puxi Scientific Instruments, Beijing, China).

Cell cycle assay. To analyze cell cycle distribution, IMR-32 and SH-SY5Y cells $\left(1 \times 10^{6}\right)$ were quickly fixed by methanol $\left(4^{\circ} \mathrm{C}\right)$ and treated with $50 \mu \mathrm{g} / \mathrm{ml}$ propidium iodide (PI) (Thermo Fisher Scientific, USA) for $20 \mathrm{~min}$. A FACSCalibur ${ }^{\mathrm{TM}}$ flow cytometer (Thermo Fisher Scientific) was used to obtain the cell cycle histograms. MultiCycle AV software (Phoenix Flow System, USA) was then used to calculate the percentages of cells in the G0/G1, S or G2/M phases.

Wound healing assay. IMR-32 and SH-SY5Y cells were seeded into 12 -well plates $\left(7.5 \times 10^{5} /\right.$ well). A sterile $1,000-\mu 1$ pipette tip was used to create the wound along the diameter of the well. The detached cells were removed, and NB cultures were maintained for $24 \mathrm{~h}$. Phase contrast images aimed at the center of the wound were captured at 0 and $24 \mathrm{~h}$ after wound creation.

Cisplatin sensitivity assay. IMR-32 and SH-SY5Y cells were seeded in 96 -well plates $\left(7.5 \times 10^{3} /\right.$ well $)$. Various concentrations of cisplatin $(0,10,25,50$ and $100 \mu \mathrm{M})$ were added into the NB culture for $24 \mathrm{~h}$. Cell survival was estimated through an MTT assay.

In vivo tumor growth assay. To evaluate the in vivo growth of NB tumors, 2-month-old female nude mice were subcutaneously inoculated in the right flank with either miR141-mimic- or C-miRNA-transduced IMR-132 cells $\left(1 \times 10^{6}\right)$. The lengths and widths of the subcutaneous tumors were measured weekly, and the tumor volume (V) was calculated using the formula: $\mathrm{V}=$ length $\mathrm{x}$ width ${ }^{2} / 2$. Five weeks later, the mice were sacrificed. Subcutaneous tumors were extracted and formalin-fixed and paraffin-embedded sections were prepared. Immunohistochemistry was then performed using an anti-Ki-67 antibody (Cell Signaling, USA).

Luciferase reporter assay. Wild-type (WT) human FUS 3'-UTR and mutated (MU) FUS 3'-UTR (with a MU sequence on the miR-141 binding site) were amplified from a human brain cDNA library and inserted between the XhoI/NotI restrictive sites of a firefly/Renilla luciferase reporter pmiRREPORT (RiboBio, Guangzhou, China). Human HEK293T cells were co-transfected with $25 \mathrm{ng} / \mathrm{ml}$ of either the luciferase reporter with WT FUS 3'-UTR (WT-3UTR), or the luciferase reporter with MU FUS 3'-UTR (MU-3UTR), and 100 pmol of either miR-141-mimics or C-miRNA. Forty-eight hours after co-transfection, a Dual-Luciferase Reporter Assay (Promega, Madison, WI, USA) was carried out according to 
the manufacturer's protocol. For each measurement, the relative firefly luciferase activity was normalized to Renilla with transfection of C-miRNA.

FUS downregulation assay. A small-interfering RNA (siRNA) against the human FUS gene (FUS-siRNA), and its control siRNA (C-siRNA) were purchased from RiboBio. In the NB culture, IMR-32 and SH-SY5Y cells were transfected with 100 nM FUS-siRNA or C-siRNA. Forty-eight hours after transfection, healthy cells were subject to qRT-PCR examination to verify the efficacy of the downregulation.

Statistical analysis. All experiments were carried out in biological triplicates. The results are presented as mean \pm standard error. SPSS 11.0 software (SPSS, Inc., Chicago, IL, USA) was applied for statistical analysis, and the Student's t-test was performed to compare the results. A statistically significant difference was defined as $\mathrm{P}<0.05$.

\section{Results}

miR-141 is downregulated in human NB cell lines. In the present study, we used qRT-PCR to determine the expression profiles of miR-141 in human NB cell lines. As compared to the expression level in the control cell line, human retinal-pigmented epithelial cells immortalized with telomerase reverse transcriptase (hTERT-RPE1), we found that miR-141 was predominantly downregulated in both the MYCN-amplified NB cell lines, IMR-32, NB-1691 and LAN-5, as well as the non-MYCN-amplified NB cell lines, SH-SY5Y, SK-N-AS and LAN-6 (Fig. 1; $\mathrm{P}<0.05$ ).

Upregulation of miR-141 inhibits the proliferation and cell cycle transition in NB cells. We infected two NB cell lines, an $M Y C N$-amplified NB cell line IMR-32 and a non$M Y C N$-amplified NB cell line SH-SY5Y, with either a lentivirus of human mature miR-141 mimics (miR-141-mimics) or a lentivirus of control miRNA mimics (C-miRNA). After lentiviral transduction was allowed to stabilize for 48 or $72 \mathrm{~h}$, qRT-PCR demonstrated that miR-141 gene expression levels were markedly upregulated in both the IMR-32 and SH-SY5Y cells by lentiviral transduction of miR-141-mimics (Fig. 2A; $\mathrm{P}<0.05)$.

We then sought to ascertain the functional mechanism of miR-141 upregulation in NB. Firstly, through a proliferation MTT assay, we found that, in both IMR-32 and SH-SY5Y cells, cell proliferation was significantly decreased by miR-141 upregulation (Fig. 2B; P<0.05). Secondly, through a cell cycle assay, we found that, in both the IMR-32 and SH-SY5Y cells, miR-141 upregulation induced significant cell cycle arrest at the $\mathrm{G} 0 / \mathrm{G} 1$ phase (Fig. $2 \mathrm{C} ; \mathrm{P}<0.05$ ).

Upregulation of miR-141 reduces migration and increases cisplatin sensitivity in NB cells. As metastatic potential and sensitivity to chemotherapeutic drugs affect the prognosis of $\mathrm{NB}$, we then sought the functional mechanism of miR-141 upregulation on NB migration and cisplatin sensitivity. Through a wound healing assay, we found that the migration capability was significantly reduced by miR-141 upregulation in the IMR-32 and SH-SY5Y cells (Fig. 3A). In a cisplatin

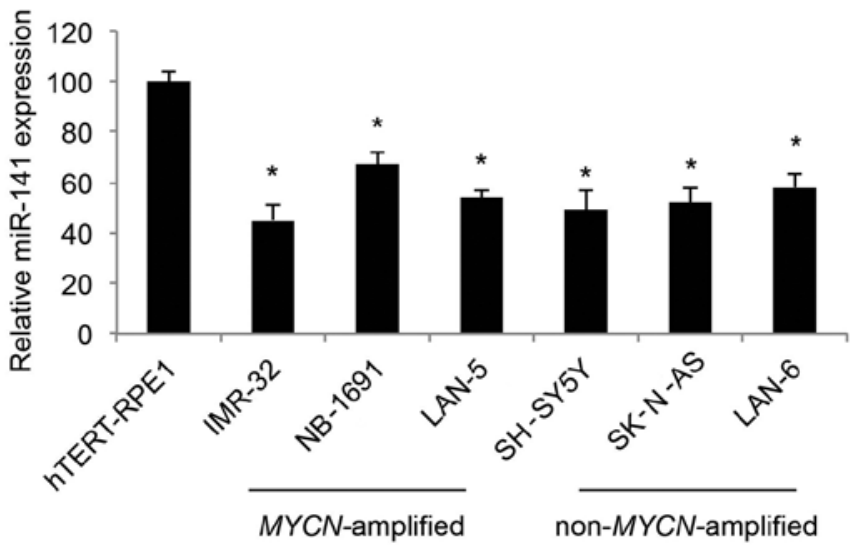

Figure 1. miR-141 is downregulated in both $M Y C N$ - and non-MYCN-amplified neuroblastoma cell lines. Expression of miR-141 was compared by qRT-PCR between a control cell line, human retinal-pigmented epithelial cells immortalized with telomerase reverse transcriptase (hTERT-RPE1), and both MYCN-amplified NB cell lines, IMR-32, NB-1691 and LAN-5, as well as non- $M Y C N$-amplified NB cell lines, SH-SY5Y, SK-N-AS and LAN-6 $\left({ }^{*} \mathrm{P}<0.05\right.$ vs. hTERT-RPE1).

chemosensitivity assay, we applied various concentrations of cisplatin to lentiviral-infected IMR-32 and SH-SY5Y cells. We found that, in response to moderate to high concentrations of cisplatin (10-100 $\mu \mathrm{M})$, miR-141 upregulation significantly increased the chemosensitivity of the IMR-32 and SH-SY5Y cell lines (Fig. 3B; $\mathrm{P}<0.05$ ).

Upregulation of miR-141 inhibits in vivo NB tumor growth. We also investigated the mechanism of miR-141 upregulation on the in vivo growth of NB tumors. miR-141-mimic- or C-miRNAinfected IMR-132 cells $\left(1 \times 10^{6}\right.$ cells) were subcutaneously injected into 2-month-old female nude mice. In vivo tumor volumes were compared between the C-miRNA-infected IMR-132 transplantations and the miR-141-mimic-infected IMR-132 transplantations every week for 5 weeks. The comparison demonstrated that, starting from 2 weeks after transplantation, in vivo NB tumor growth was significantly inhibited by miR-141 upregulation (Fig. 4A; $\mathrm{P}<0.05$ ). Five weeks after transplantation, the carrier mice were sacrificed. NB tumors were extracted, formalin-fixed and prepared was paraffin-embedded sections. Immunohistochemistry with the Ki-67 antibody confirmed that the in vivo proliferation of IMR-32 cells was significantly inhibited by miR-141 upregulation (Fig. 4B).

FUS is inversely associated with miR-141 in NB cells. We then sought the possible downstream signaling pathway of miR-141 in NB. We searched some of the miRNA targeting algorithms, including microRNA.org (www.microrna.org) and TargetScanHuman (www.targetscan.org). It was noted that FUS is a candidate gene with a complimentary miR-142 binding site on its 3'-UTR (Fig. 5A). A luciferase activity assay confirmed this hypothesis by showing that WT FUS 3'-UTR was regulated by miR-141, whereas MU FUS 3'-UTR (without miR-141 binding sequences) was not (Fig. 5B; $\mathrm{P}<0.05, \mathrm{P}>0.05)$. 
A

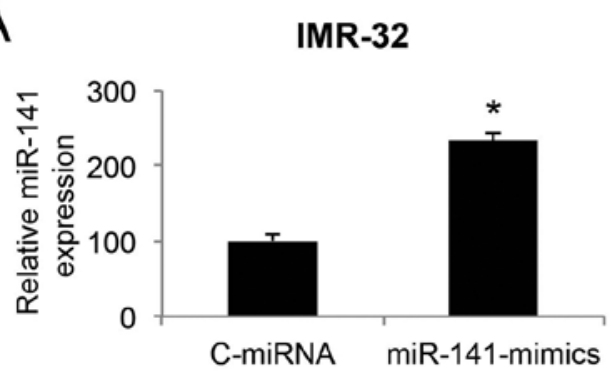

B

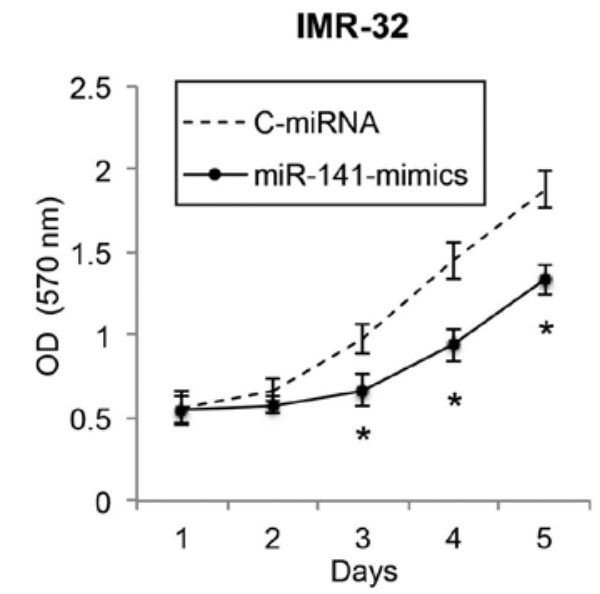

C

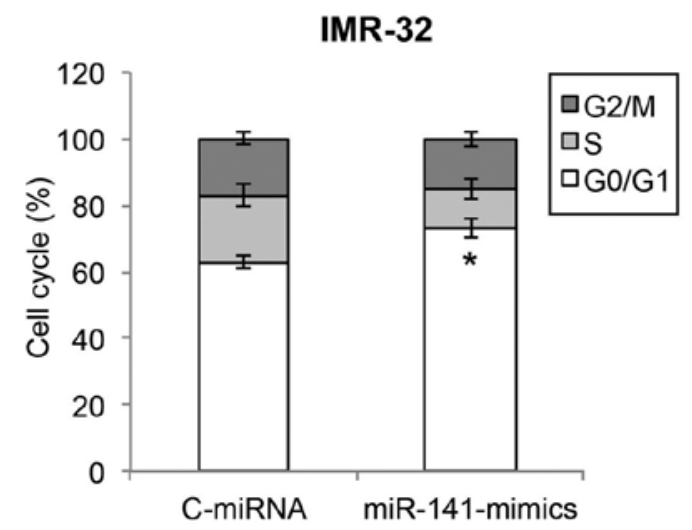

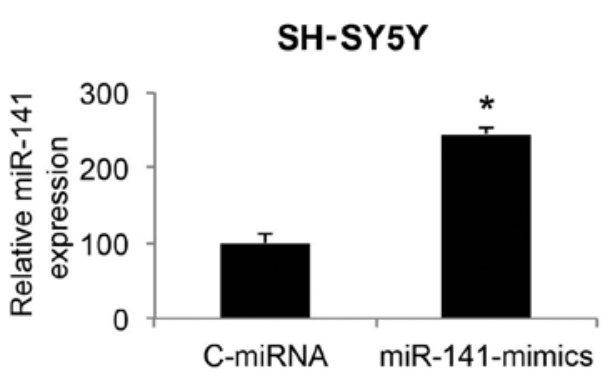

SH-SY5Y

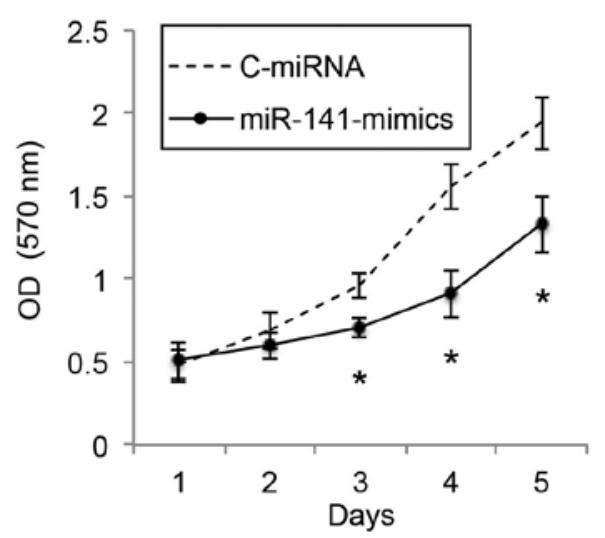

SH-SY5Y

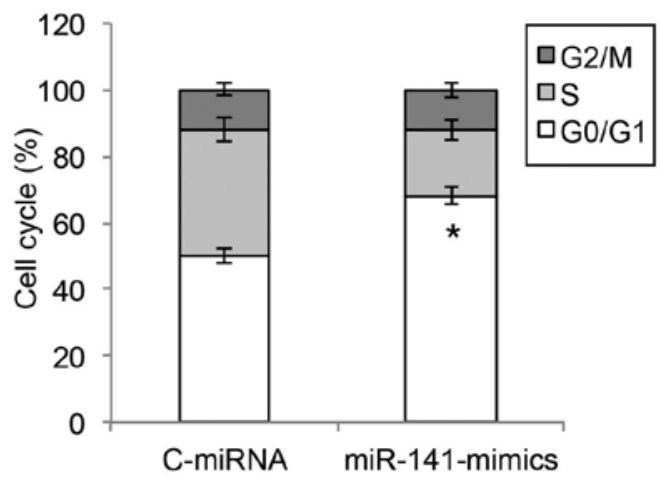

Figure 2. miR-141 upregulation inhibits proliferation and cell cycle progression in neuroblastoma cell lines. NB cell lines, IMR-32 and SH-SY5Y, were infected with a miRNA-141 mimic lentivirus to upregulate the endogenous expression level of miR-141 (miR-141-mimics). The control NB cells were infected with a control miRNA lentivirus (C-miRNA). (A) qRT-PCR was used to compare the endogenous miR-141 expression between NB cells infected with C-miRNA and NB cells infected with miR-141-mimics ("P<0.05). (B) An MTT assay was used for 5 days to compare cell proliferation ability between the NB cells infected with C-miRNA and the NB cells infected with miR-141-mimics ("P<0.05). (C) A cell cycle assay was used to compare the percentages of G0/G1-, S- and G2/M- phase cells infected with C-miRNA and those infected with miR-141-mimics ("P<0.05).

We investigated the gene expression pattern of FUS in NB cell lines. The results of qRT-PCR showed that FUS was upregulated in both the $M Y C N$ - and non- $M Y C N$-amplified NB cells, as compared to the control cell line hTERT-RPE1 (Fig. 5C; $\mathrm{P}<0.05)$. Thus, the expression levels of FUS and miR-141 are inversely correlated in NB cell lines.

We then examined whether FUS expression was affected by miR-141 upregulation, by comparing the qRT-PCR results between NB cells transduced with C-miRNA and NB cells transduced with miR-141-mimics. The results demonstrated that, in both the IMR-32 and SH-SY5Y cell lines, FUS was inversely regulated, or downregulated by miR-141 upregulation (Fig. 5D; $\mathrm{P}<0.05$ ).

Downregulation of FUS inhibits the proliferation and cell cycle transition in NB cells. Since FUS was found to be inversely correlated with miR-141 in NB, we aimed to ascertain whether downregulation of FUS would have similar tumor-suppressive effects as miR-141 upregulation in NB. We transfected IMR-32 and SH-SY5Y cells with either the 
A

IMR-32

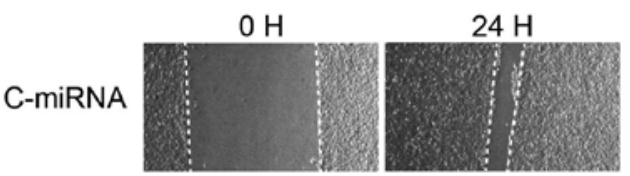

miR-141-mimics
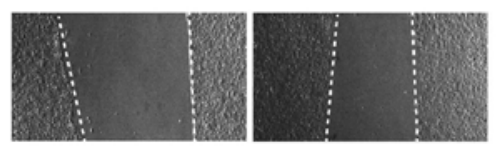

B

IMR-32

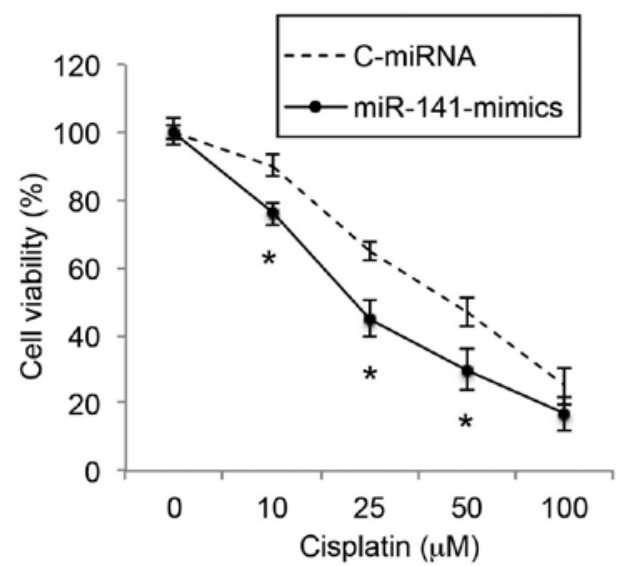

SH-SY5Y
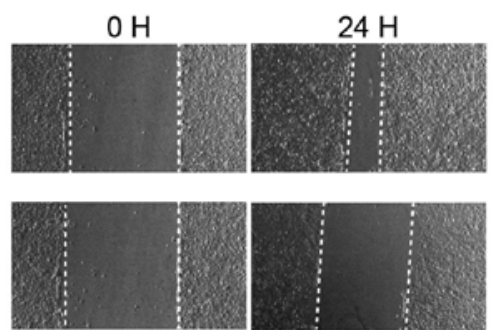

SH-SY5Y

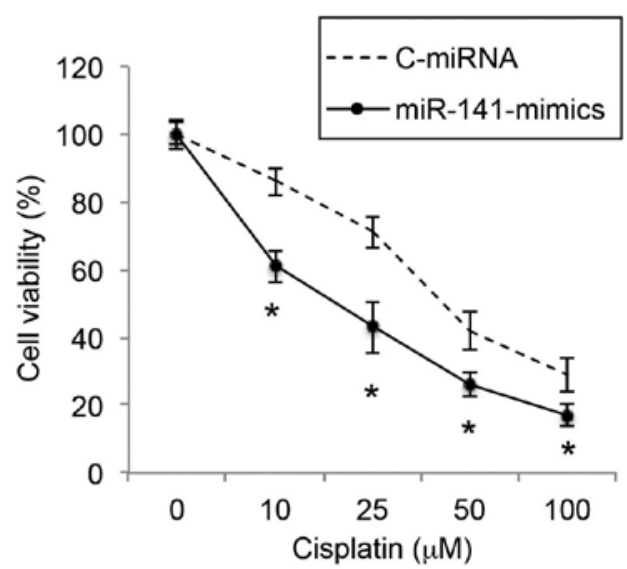

Figure 3. miR-141 upregulation inhibits migration and increases cisplatin sensitivity in neuroblastoma cell lines. (A) A wound healing assay was used to compare the migratory capability of the NB cells infected with C-miRNA and the NB cells infected with miR-141-mimics. Phase contrast images were captured at 0 and $24 \mathrm{~h}$ after an initial wound was created on a cell monolayer. (B) After lentiviral infection, IMR-32 and SH-SY5Y cells were incubated with various concentrations of cisplatin at $0,10,25,50$ or $100 \mu \mathrm{M}$ for $24 \mathrm{~h}$. An MTT assay was used to compared the cisplatin sensitivity between NB cells infected with $\mathrm{C}$-miRNA and NB cells infected with miR-141-mimics ( $\mathrm{P}<0.05)$.

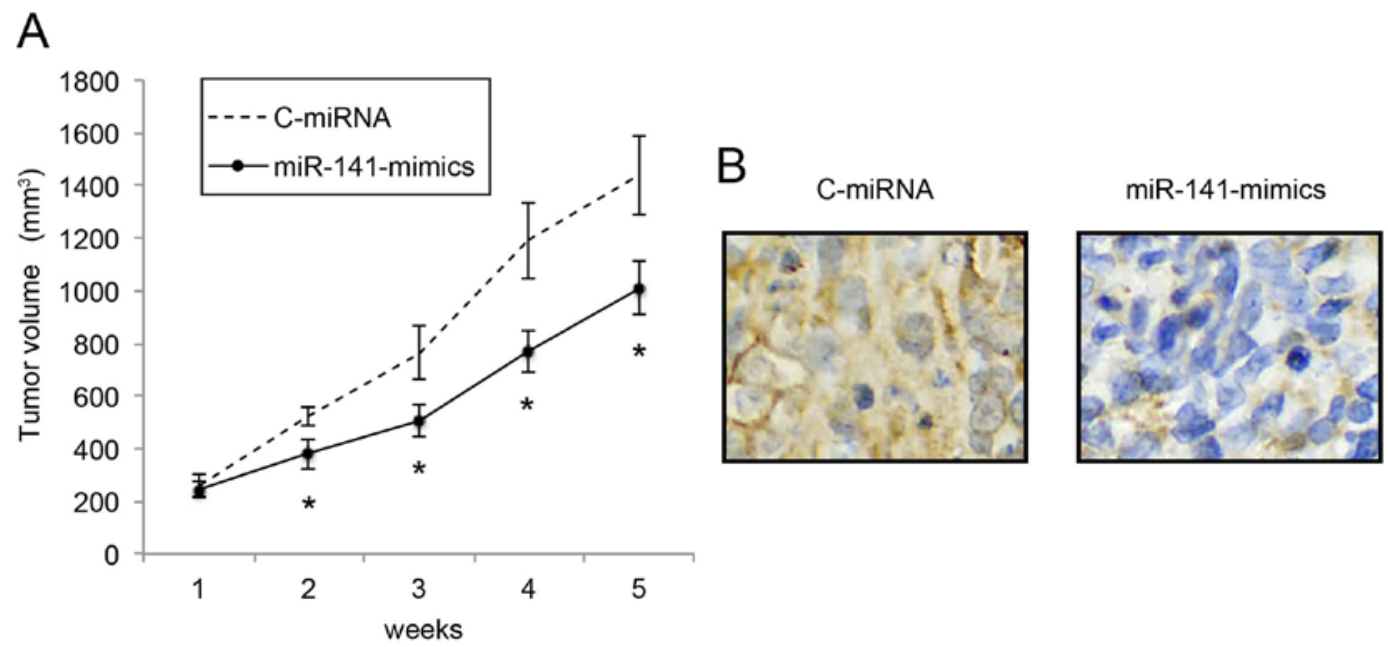

Figure 4. miR-141 upregulation inhibits the in vivo growth of neuroblastoma transplanted tumors. (A) Two month-old female nude mice were subcutaneously inoculated in the right flank with either miR-141-mimic- or C-miRNA-transduced IMR-132 cells $\left(1 \times 10^{6}\right)$. For 5 weeks, in vivo tumor volumes were weekly compared between miR-141-mimic- and C-miRNA-transduced IMR-132 transplantations ( $\mathrm{P}<0.05$ ). (B) Five weeks after transplantation, NB tumors were extracted and prepared in formalin-fixed and paraffin-embedded sections, followed by Ki-67 immunostaining.

FUS-targeted siRNA (FUS-siRNA) or a control siRNA (C-siRNA). Results of qRT-PCR showed that the FUS level was markedly downregulated by FUS-siRNA in both the IMR-32 and SH-SY5Y cells (Fig. 6A; P<0.05). Based on a 
A

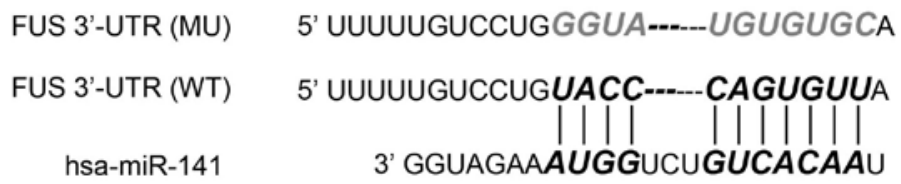

B

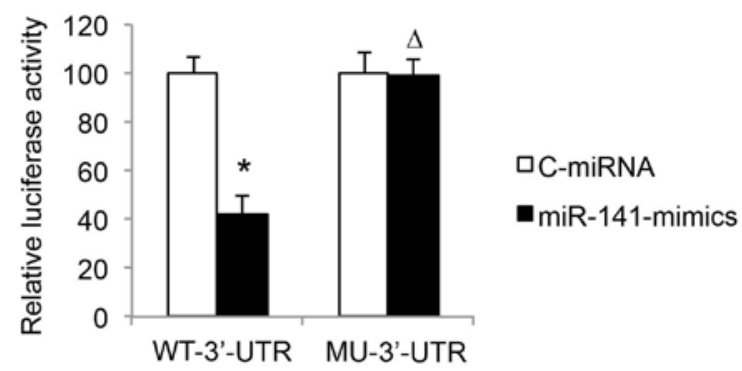

C

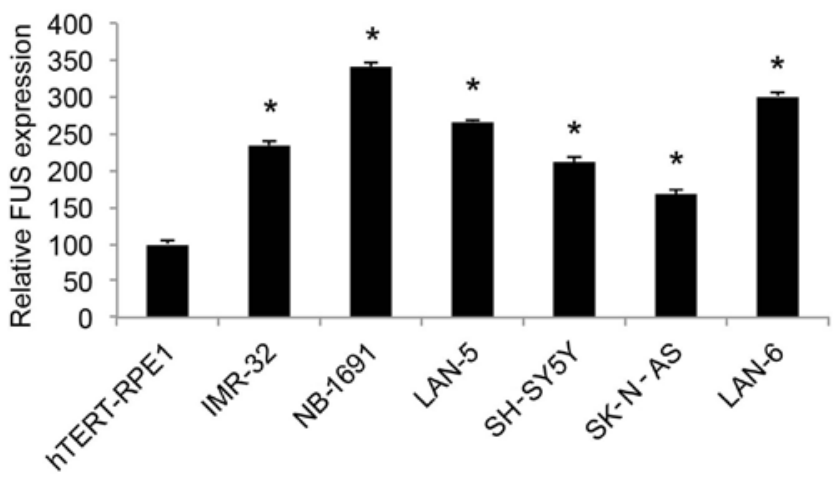

D

IMR-32

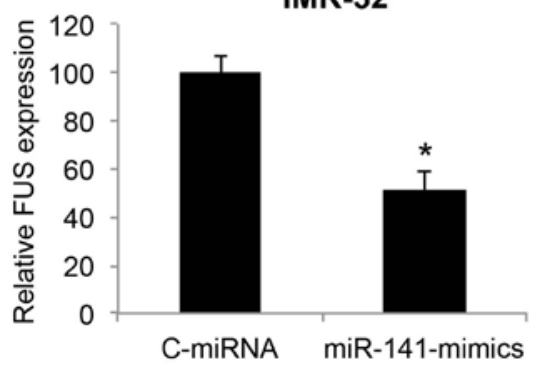

SH-SY5Y

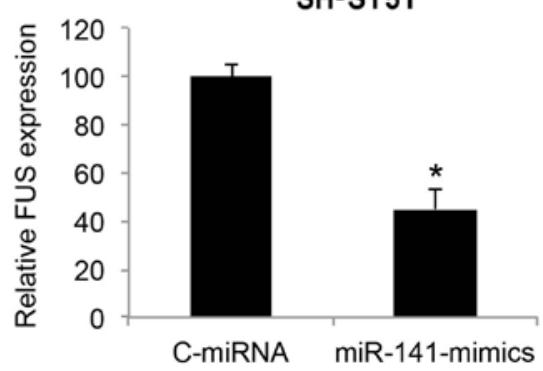

Figure 5. FUS is inversely regulated by miR-141 in neuroblastoma cell lines. (A) The complimentary binding of human miR-141 on the 3'-UTR of the wild-type (WT) FUS gene is highlighted. The binding sequence was also mutated (MU) to deactivate the binding of miR-141. (B) Wild-type FUS 3'-UTR (WT-3UTR) and MU FUS 3'-UTR (MU-3UTR) were inserted into pmiR-REPORT luciferase vector, and co-transfected with miR-141-mimics or C-miRNA into HEK293T cells, respectively. Forty-eight fours after co-transfection, relative luciferase activity was characterized by a dual-luciferase reporter assay $\left({ }^{*} \mathrm{P}<0.05 ;{ }^{\Delta} \mathrm{P}>0.05\right)$. (C) FUS expression was compared by qRT-PCR between hTERT-RPE1, and both $M Y C N$-amplified NB cell lines, IMR-32, NB-1691 and LAN-5, as well as non-MYCN-amplified NB cell lines, SH-SY5Y, SK-N-AS and LAN-6 ("P<0.05, vs. hTERT-RPE1). (D) FUS expression was compared by qRT-PCR between NB cells infected with C-miRNA and NB cells infected with miR-141-mimics ( $\mathrm{P}<0.05)$.

proliferation MTT assay, we found that, in both IMR-32 and SH-SY5Y cells, cell proliferation was significantly decreased by FUS downregulation (Fig. 6B; $\mathrm{P}<0.05$ ). In addition, through a cell cycle assay, we found that FUS downregulation induced significant cell cycle arrest at the G0/G1 phase in the NB cells (Fig. 6C; $\mathrm{P}<0.05$ ). Thus, FUS downregulation showed similar inhibitory effects as miR-141 upregulation in regards to cell proliferation and cell cycle arrest in the NB cell lines.

Downregulation of FUS reduces migration and increases the cisplatin sensitivity in NB cells. Finally, we investigated the effects of FUS downregulation on NB migration and cisplatin sensitivity. In a wound healing assay, we found that the cell migratory capability was significantly reduced by FUS downregulation in both the IMR-32 and SH-SY5Y cells (Fig. 7A). In the cisplatin chemosensitivity assay, we also found that FUS downregulation significantly increased cisplatin sensitivity in the NB cells (Fig. 7B; $\mathrm{P}<0.05$ ). Thus, our data demonstrated that FUS downregulation also had tumor-suppressive effects similar to those of miR-141 upregulation on cancer metastasis and chemosensitivity in the NB cells. 
A

IMR-32

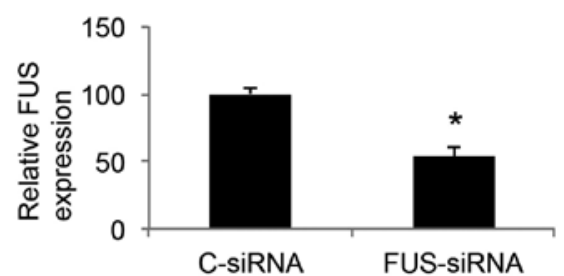

B

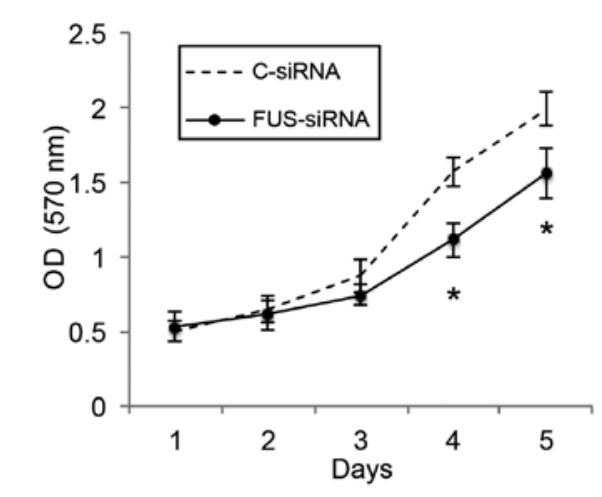

C

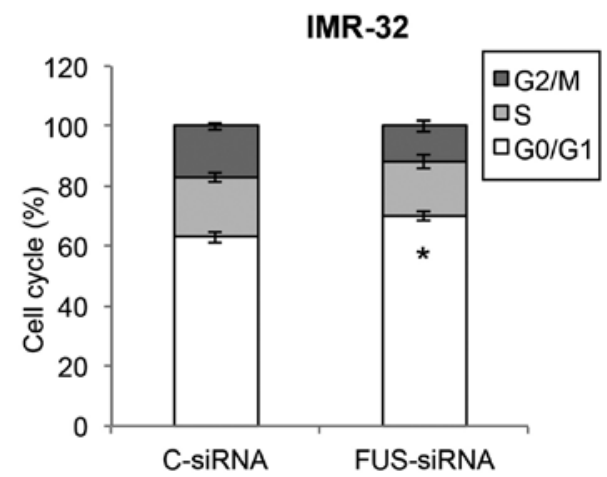

B

IMR-32

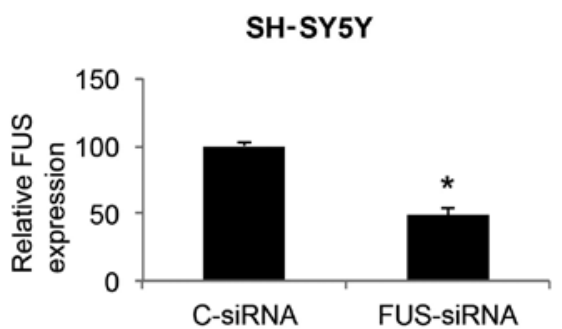

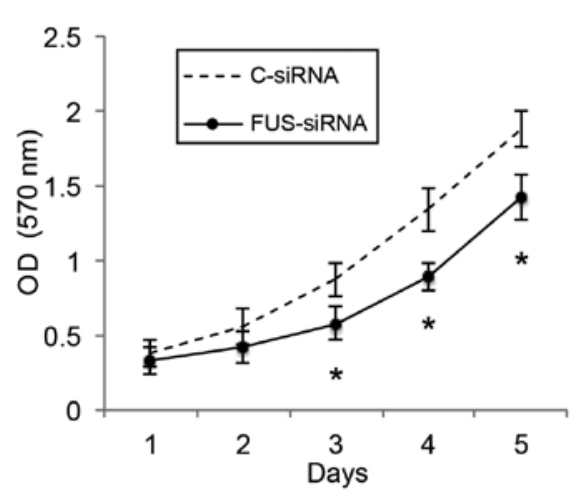

SH-SY5Y

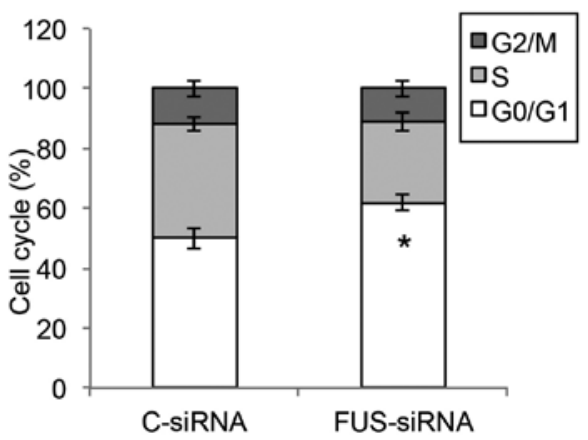

Figure 6. FUS downregulation inhibits proliferation and cell cycle progression in neuroblastoma cell lines. IMR-32 and SH-SY5Y cells were transfected with FUS-specific siRNA (FUS-siRNA) or control siRNA (C-siRNA). (A) qRT-PCR was used to compare the endogenous FUS expression between NB cells transfected with FUS-siRNA and NB cells transfected with C-siRNA ("P<0.05). (B) An MTT assay was used to compare cell proliferation ability between NB cells transfected with FUS-siRNA and NB cells transfected with C-siRNA ("P<0.05). (C) A cell cycle assay was used to compare the percentages of G0/G1-, S- and G2/M-phase NB cells transfected with FUS-siRNA and those of the NB cells transfected with C-siRNA ("P<0.05).

\section{Discussion}

In human neuroblastoma (NB), miRNAs have been shown to play important roles as both biomarkers for prediction of prognosis and as genetic modulators for regulation of cancer development $(7,17)$. In the present study, we demonstrated that miR-141, a cancer-associated miRNA that has never been characterized in NB before the present study, was universally downregulated in both $M Y C N$ - and non- $M Y C N$-amplified NB cell lines. The functional role of miR-141 in NB was investigated by lentiviral transduction assay, in which miR-141 was stably overexpressed in NB cell lines IMR-32 and SH-SY5Y. miR-141 was found to negatively regulate NB development, including both in vitro and in vivo proliferation, cell cycle progression, migration and cisplatin chemosensitivity. These results strongly suggest a tumor-suppressive role of miR-141 in NB. The tumor-suppressive effect of miR-141 has been shown in other types of cancers. In gastric cancer, miR-141 was found to be significantly downregulated in both human tumors and gastric cancer cell lines, and upregulation of miR-141 significantly inhibited the tumor growth in gastric cancer cell lines (10). Notably, in other types of human cancer, miR-141 may also exert an oncogenic effect. For example, high expression of serum miR-141 was shown to be closely associated with patients with advanced colon cancer (9). Thus, whether miR-141 acts as an oncogene or a tumor-suppressor gene is largely dependent on the host cancer type, and possibly the downstream signaling pathways associated with miR-141.

We extended the present study to ascertain the target gene of miR-141, and revealed that the FUS gene was inversely correlated with miR-141. Luciferase reporter assay demonstrated that 3'-UTR of FUS was attached by miR-141. qRT-PCR 
A

IMR-32

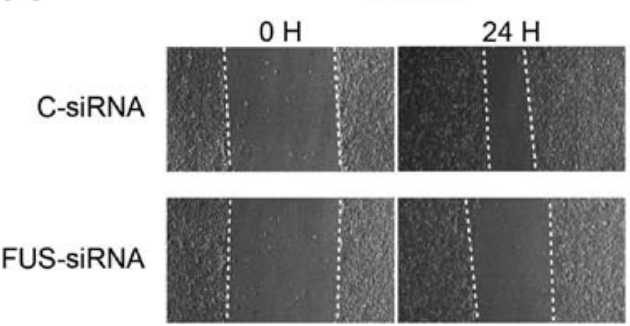

SH-SY5Y
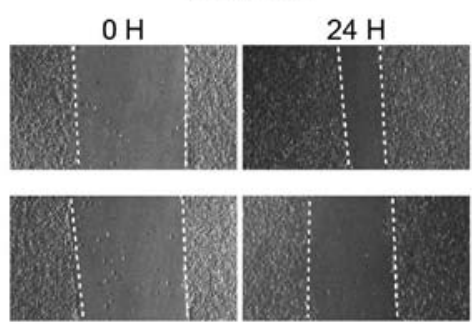

B
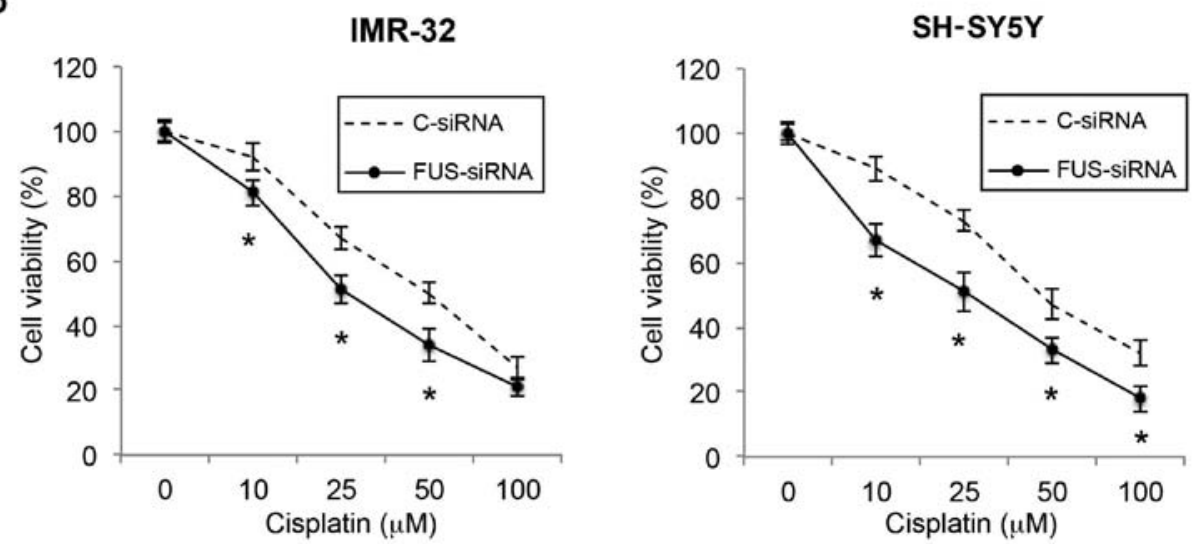

Figure 7. FUS downregulation inhibits migration and increases cisplatin sensitivity in neuroblastoma cell lines. (A) A wound healing assay was used to compare the migratory capability between NB cells transfected with FUS-siRNA and NB cells transfected with C-siRNA. Phase contrast images were captured at 0 and $24 \mathrm{~h}$ after initial wounding. (B) After siRNA transfection, IMR-32 and SH-SY5Y cells were incubated with various concentrations of cisplatin at $0,10,25,50$ or $100 \mu \mathrm{M}$ for $24 \mathrm{~h}$. An MTT assay was used to compare the cisplatin sensitivity between NB cells transfected with FUS-siRNA and NB cells transfected with C-siRNA ( $\mathrm{P}<0.05)$.

assay showed that miR-141 upregulation downregulated FUS in the IMR-32 and SH-SY5Y cells. Most importantly, in vitro functional assays showed that siRNA-induced FUS downregulation had similar tumor-suppressive effects as those noted by miR-141 upregulation on NB proliferation, cell cycle progression, metastasis and chemosensitivity. Based on these data, miR-141 and FUS are inversely associated, not only in terms of expression pattern but also concerning the functional regulation on NB development. Thus, miR-141 is a tumor suppressor, whereas FUS is an oncogene in NB.

Notably, in other types of cancer, such as prostate cancer, FUS acts as a tumor-suppressor gene and inhibits cancer proliferation and induces apoptosis (15). The disparity in its oncogenic vs. tumor-suppressive role in different cancer types may also be attributed to the complex molecular pathways associated with FUS regulation in different cancer types. Therefore, experiments to explore the possible signaling pathways associated with FUS in NB would be extremely helpful in deciphering the underlying mechanisms of NB pathogenesis. Notably, the direct correlation of miR-141 and FUS on their inverse effects on NB has yet to be established. Thus, further experiments investigating FUS overexpression under the circumstance of miR-141 upregulation are warranted, as it will shed light on whether FUS may ameliorate the tumor suppressive functions in NB induced by miR-141 upregulation.

In conclusion, we demonstrated a novel signaling pathway of tumor-suppressor miR-141, inversely associated with oncogene FUS, in human NB. The findings of the present study may help identify new biomarkers or therapeutic targets for patient with neuroblastoma.

\section{References}

1. Brodeur GM: Neuroblastoma: Biological insights into a clinical enigma. Nat Rev Cancer 3: 203-216, 2003.

2. Siegel RL, Miller KD and Jemal A: Cancer statistics, 2015. CA Cancer J Clin 65: 5-29, 2015.

3. Brodeur GM and Fong CT: Molecular biology and genetics of human neuroblastoma. Cancer Genet Cytogenet 41: 153-174, 1989.

4. Corvi R, Amler LC, Savelyeva L, Gehring M and Schwab M: MYCN is retained in single copy at chromosome 2 band p23-24 during amplification in human neuroblastoma cells. Proc Natl Acad Sci USA 91: 5523-5527, 1994.

5. Tsuda T, Obara M, Hirano H, Gotoh S, Kubomura S, Higashi K, Kuroiwa A, Nakagawara A, Nagahara N and Shimizu K: Analysis of $\mathrm{N}-m y c$ amplification in relation to disease stage and histologic types in human neuroblastomas. Cancer 60: 820-826, 1987.

6. Alvarez-Garcia I and Miska EA: MicroRNA functions in animal development and human disease. Development 132: 4653-4662, 2005.

7. Schulte JH, Horn S, Schlierf S, Schramm A, Heukamp LC, Christiansen H, Buettner R, Berwanger B and Eggert A: MicroRNAs in the pathogenesis of neuroblastoma. Cancer Lett 274: 10-15, 2009.

8. Das S, Bryan K, Buckley PG, Piskareva O, Bray IM, Foley N, Ryan J, Lynch J, Creevey L, Fay J, et al: Modulation of neuroblastoma disease pathogenesis by an extensive network of epigenetically regulated microRNAs. Oncogene 32: 2927-2936, 2013.

9. Cheng H, Zhang L, Cogdell DE, Zheng H, Schetter AJ, Nykter M, Harris CC, Chen K, Hamilton SR and Zhang W: Circulating plasma miR-141 is a novel biomarker for metastatic colon cancer and predicts poor prognosis. PLoS One 6: e17745, 2011. 
10. Du Y, Xu Y, Ding L, Yao H, Yu H, Zhou T and Si J: Downregulation of miR-141 in gastric cancer and its involvement in cell growth. J Gastroenterol 44: 556-561, 2009.

11. Yaman Agaoglu F, Kovancilar M, Dizdar Y, Darendeliler E, Holdenrieder S, Dalay N and Gezer U: Investigation of miR-21, miR-141, and miR-221 in blood circulation of patients with prostate cancer. Tumour Biol 32: 583-588, 2011.

12. Chen Y and Stallings RL: Differential patterns of microRNA expression in neuroblastoma are correlated with prognosis, differentiation, and apoptosis. Cancer Res 67: 976-983, 2007.

13. Vance C, Rogelj B, Hortobágyi T, De Vos KJ, Nishimura AL, Sreedharan J, Hu X, Smith B, Ruddy D, Wright P, et al: Mutations in FUS, an RNA processing protein, cause familial amyotrophic lateral sclerosis type 6. Science 323: 1208-1211, 2009.

14. Kwiatkowski TJ Jr, Bosco DA, Leclerc AL, Tamrazian E, Vanderburg CR, Russ C, Davis A, Gilchrist J, Kasarskis EJ, Munsat T, et al: Mutations in the FUS/TLS gene on chromosome 16 cause familial amyotrophic lateral sclerosis. Science 323 1205-1208, 2009.
15. Brooke GN, Culley RL, Dart DA, Mann DJ, Gaughan L, McCracken SR, Robson CN, Spencer-Dene B, Gamble SC, Powell SM, et al: FUS/TLS is a novel mediator of androgendependent cell-cycle progression and prostate cancer growth. Cancer Res 71: 914-924, 2011.

16. Khursheed K, Wilm TP, Cashman C, Quinn JP, Bubb VJ and Moss DJ: Characterisation of multiple regulatory domains spanning the major transcriptional start site of the FUS gene, a candidate gene for motor neurone disease. Brain Res 1595: 1-9, 2015.

17. Lin RJ, Lin YC, Chen J, Kuo HH, Chen YY, Diccianni MB, London WB, Chang $\mathrm{CH}$ and Yu AL: microRNA signature and expression of Dicer and Drosha can predict prognosis and delineate risk groups in neuroblastoma. Cancer Res 70: 7841-7850, 2010 